\title{
ASSESSING HAZARD PROBABILITY FACTORS RELATED TO FORECASTED WEATHER CONDITIONS
}

\author{
V.G. Burmistrova ${ }^{1}$, A.A Butov ${ }^{1}$, A.V. Zharkov ${ }^{1}$, Yu.V. Pchelkina ${ }^{2}$ \\ ${ }^{1}$ Ulyanovsk State University, Ulyanovsk, Russia \\ ${ }^{2}$ Samara National Research University, Samara, Russia
}

\begin{abstract}
In this paper we have considered two meteorological factors as an object of our research: horizontal and vertical visibility. These meteorological factors are associated with "danger thresholds", going beyond these levels (in excess or decrease) is unacceptable or undesirable. The initial values of the horizontal and vertical visibility are assumed to be equal to the values presented in the weather forecast. The results of this development can be used to solve problems related to aviation forecasting, for example, when making a decision regarding a departure or landing of the aircraft.
\end{abstract}

Keywords: meteorological conditions, assessment of probability, modeling, forecast.

Citation: Burmistrova VG, Butov AA, Zharkov AV, Pchelkina YuV. Assessing hazard probability factors related to forecasted weather conditions. CEUR Workshop Proceedings, 2016; 1638: 521-526. DOI: 10.18287/16130073-2016-1638-521-526

\section{Introduction}

In this paper we consider the weather report, which is transmitted in the form of reports METAR (Meteorological Aerodrome Report — aeronautical meteorological code for transmitting reports on the actual weather at the airport) or TAF (the weather at a certain time in the future (normally from several hours to days).

The forecast TAF can be represented in the form of one or more sections, each of which begins with one of the code words:

NOSIG (No significant change) - this means that there are no major weather changes in the next 2 hours;

BECMG (Becoming) - sustained significant changes of weather conditions are expected;

TEMPO (Temporary) - temporary significant changes of weather conditions are expected.

The values of the studied meteorological factors are modeled based on the justification of the forecast (forecast errors and probability). We took into account not only 
the bulk of the weather forecast but also a group of stable and temporary changes in the forecast. The main objective of this work is to determine the probability of the value of meteorological factors to go beyond the "hazard threshold".

The solution to this problem has been found under the assumption that specific implementation of meteorological factors at the time of landing (or take off) is a continuous random variable with values on a usual quantitative scale, and it has a normal distribution and that the meteorological factors are independent variables.

We will consider $\mathrm{X}(\mathrm{t})$ to be some meteorological factor provided in the actual weather report (METAR) or airport weather forecast (TAF). It is a continuous onedimensional value (a mark on a quantitative scale) by which we will consider parameters of meteorological factors: horizontal visibility (in meters) at approaching of the aircraft or the height of the lower threshold of the cloud (in meters) at the terminal aerodrome [1].

The value of meteorological factor $\mathrm{X}(\mathrm{t})$ can be provided in the main part of the forecast (defined as $X_{\text {forecast }}$ ), and in the group of persistent changes (they are defined as BECMG, FM), or in the group of temporal changes (defined as TEMPO), sometimes indicating the probability of possible changes in 40\% (PROB40) or 30\% (PROB30).

The value of meteorological factors not included into the main part of the weather forecast will be defined as $X_{\text {tempo }}$.

"Maximum hazard threshold" is associated with these meteorological factors - the value of going beyond it (the excess or decrease) is unacceptable or undesirable, the value of this level of "maximum danger level" is defined by [2]-[6].

The basic assumption of this article is the following: the definite implementation of meteorological factor $\mathrm{X}(\mathrm{t})$ at the time of landing (or take off) is a continuous random variable with values in the usual quantitative scale.

Our main problem is to find probability of the event that the value $\mathrm{X}(\mathrm{t})$ value will overcome $X_{\text {danger }}$, defined as

$P=P\left\{X(t)>X_{\text {danger }}\right\}$ or $P=P\left\{X(t)<X_{\text {danger }}\right\}$.

We define $P$ as the probability of the weather conditions exceeding the safety threshold.

We will assume that:

- distribution of possible $X(t)$ implementations are Gaussian (normal) with parameters $a$ and $\sigma$, [7] - [8];

- the value $X_{\text {forecast }}$ is the value of the parameter $a$ (i.e., the mean value, and mode (the highest value) of the distribution density).

- the value $\sigma$ is derived from the conditions of accuracy and statistical validity of the forecast implementation.

\section{Calculating Estimated Probability of Weather Conditions}

Let us define $L(t)$ as the horizontal visibility (which can be in a real situation) and will be a random variable. The variable $L_{\text {forecast }}$ is defined in the TAF forecast sec- 
tion. The variable of the horizontal visibility $L(t) \in \mathrm{N}\left(L_{\text {forecast }} ; \sigma\right)$ has a Gaussian (normal) distribution (with mathematical expectation $L_{\text {forecast }}$ and variance $\sigma^{2}$ ). The variance of $L(t)$ is determined by the forecast provision and accuracy.

According to [9], at forecasted visibility of more than 800 meters, the error is equal to $30 \%$ of the forecast and $80 \%$ of provision. In this case the following is true:

$P\left\{\left|L(t)-L_{\text {forecast }}\right|<0.3 \cdot L_{\text {forecast }}\right\}=0.8$

Hence we can derive $\sigma=0.23 \cdot L_{\text {forecast }}$.

We obtain the probability of weather conditions exceeding the safety threshold in this situation (forecast visibility of more than $800 \mathrm{~m}$ ):

$P\left\{L(t)<L_{\text {danger }}\right\}=\Phi\left(4.27 \cdot\left(\frac{L_{\text {danger }}}{L_{\text {forecast }}}-1\right)\right)$

where $\Phi(*)$ is the function of the standard Gaussian distribution. Geometric illustration of the formula (2) is given in Figure 1.

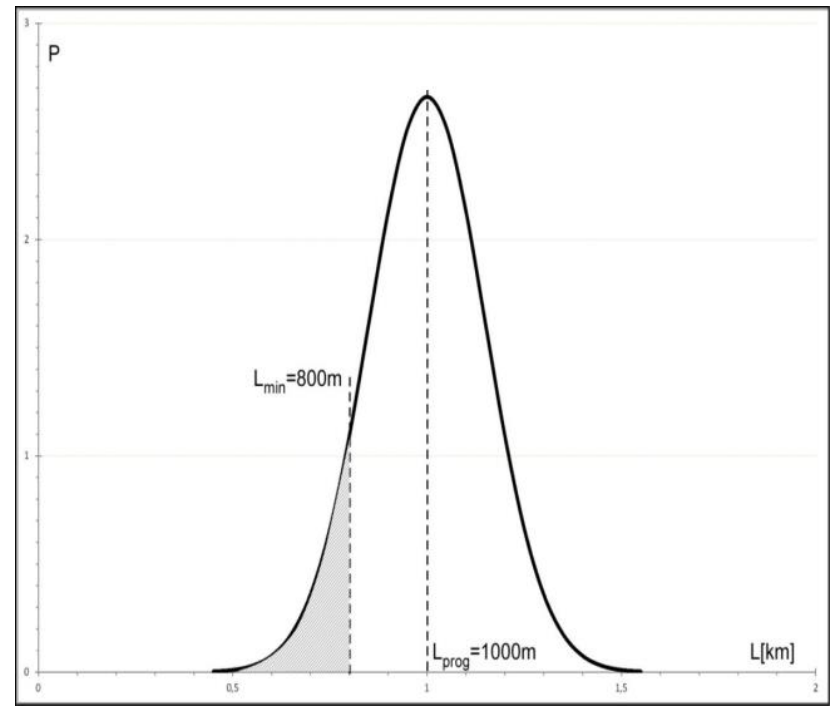

Fig. 1. Determining visibility probability is below the minimum value

If the value of the forecast visibility is less than 800 meters, then the error is equal to $\pm 200 \mathrm{~m}$ and taking into account the reliability of $80 \%$ of the forecast, the following is true:

$P=P\left\{L_{\text {forecast }}-200<L(t)<L_{\text {forecast }}+200\right\}=0.8$

Herewith we will obtain $\sigma=156 \mathrm{~m}$.

Then the probability of a "dangerous" weather situation is equal to: 
$P\left\{L(t)<L_{\text {danger }}\right\}=\Phi\left(4.27 \cdot\left(\frac{L_{\text {danger }}}{L_{\text {forecast }}}-1\right)\right)$

Let us define the observable value of the cloud conditions $H(t), H(t) \in$ $N\left(H_{\text {forecast }} ; \sigma\right)$ where $H_{\text {forecast }}$ is the value of cloud conditions provided by a forecast. Forecasted clouds height means that the error is $\pm 30 \%$ of the forecast if the forecast value lies from $300 \mathrm{~m}$ to $3 \mathrm{~km}$, and the error is $\pm 30 \mathrm{~m}$ if the forecast value lies below $300 \mathrm{~m}$.

We will suppose the forecast is below $300 \mathrm{~m}$. Given the forecast accuracy of $70 \%$, we will claim:

$P=P\left\{H_{\text {forecast }}-30<H(t)<H_{\text {forecast }}+30\right\}=0.7$

Herewith we will obtain $\sigma=29 \mathrm{~m}$.

Then the probability of a "dangerous" weather situation is equals:

$P\left\{H(t)<H_{\text {danger }}\right\}=\Phi\left(\frac{H_{\text {danger }}-H_{\text {forecast }}}{\sigma}\right)$

If the value $H_{\text {forecast }}$ is more than $300 \mathrm{~m}$, then $H_{\text {forecast }}$ is $30 \%$ for accuracy and $70 \%$ (according to [2]) and the following is true:

$P=P\left\{\left|H(t)-H_{\text {forecast }}\right|<0.3 \cdot H_{\text {forecast }}\right\}=0.7$

Herewith we will obtain $\sigma=0.29 \cdot H_{\text {forecast }}$.

The probability of a "dangerous" weather situation equals:

$P\left\{H(t)<H_{\text {danger }}\right\}=\Phi\left(3.46 \cdot\left(\frac{H_{\text {danger }}}{H_{\text {forecast }}}-1\right)\right)$

The horizontal and vertical visibilities are considered when determining the assessment of weather conditions implementations beyond the safety threshold. Let the probability of exceeding "maximum level of hazard" of the studied weather factors be defined as $P_{1}$ and $P_{2}$. Herein considered values are independent variables (as the accuracy and correctness of the forecast of each value are considered) although they are weather dependent. We will define the probability of the event as:

$P(t)=1-\left(1-P_{1}\right)\left(1-P_{2}\right)$

Taking into account weather conditions changes, TAF forecast may be an indicator of possible changes (BECMG, FM or TEMPO). If changes are not forecasted, then NOSIG indicator may emerge. In this event in TEMPO, the value $L_{\text {tempo }}$ should be obtained from the weather forecast.

The emergence of the event from TEMPO is used with the indication within period from $T_{1}$ to $T_{2}$ that is the mixture of two weather conditions at that time and in the place: one with characteristics of the main forecast, the other from the forecast of changes. The probability of encountering TEMPO weather at the time of aircraft arrival equals: 
$p_{0}=\left\{\begin{array}{l}0.5, \text { if set to TEMPO and not set to PRPOB, } \\ 0.4, \text { if set to TEMPO and set to PRPOB } \\ 0.3, \text { ifset to TEMPO and set to PRPOB } 30 .\end{array}\right.$

Accordingly, the probability of finding the weather conditions forecast indicated in the main forecast equals $\left(1-p_{0}\right)$

The BECMG forecast replaces the main forecast from time $T_{2}$ when there is an improvement of weather conditions and from time $T_{1}$ if there is deterioration in the weather conditions with the forecast reliability of $80 \%$.

For example, let the visibility distance be $L_{\text {forecast }}$ (then the real visibility is a random variable Gaussian distributed with parameters $a=L_{\text {forecast }}$ and $\sigma_{1}=\lambda$. $L_{\text {forecast }}$ ), whereas the visibility distance is $L_{\text {tempo }}$ in TEMPO forecast (i.e. the new forecasted visibility is a random value distributed with parameters $a=L_{\text {forecast }}$ and $\sigma_{1}=\lambda \cdot L_{\text {forecast }}$ ).

Then the probability of a "dangerous" weather situation is equal to:

$P=P\left\{L(t)<L_{\text {danger }}\right\}=$

$=p_{0} \cdot \Phi\left(\frac{1}{\lambda} \cdot\left(\frac{L_{\text {danger }}}{L_{\text {tempo }}}-1\right)\right)+\left(1-\mathrm{p}_{0}\right) \Phi\left(\frac{1}{\lambda} \cdot\left(\frac{L_{\text {danger }}}{L_{\text {forecast }}}-1\right)\right)$

Depending on the values $L_{\text {forecast }}, L_{\text {tempo }}$ we will select pre-adapted formulas (2) or (4).

For the vertical visibility weather conditions $H(t)$ (i.e. the clouds height) formulas are similar:

$P=P\left\{H(t)<H_{\text {danger }}\right\}=$

$=p_{0} \cdot \Phi\left(\frac{1}{\lambda} \cdot\left(\frac{H_{\text {danger }}}{H_{\text {tempo }}}-1\right)\right)+\left(1-p_{0}\right) \Phi\left(\frac{1}{\lambda} \cdot\left(\frac{H_{\text {danger }}}{H_{\text {forecast }}}-1\right)\right)$

\section{References}

1. Federal aviation regulations "Preparation and flight operations in civil aviation of the Russian Federation", 2009; 128; 95 p. [in Russian]

2. ICAO. Annex. 3 to the Chicago Convention on International Civil Aviation. Meteorological Service for International Air Navigation. Appendix B. Forecast accuracy desirable from an operational point of view, 2010; 17; $218 \mathrm{p}$.

3. Analysis of the impact of Reliability on the safety of flights by type of aircraft. M., 2009. URL: http://www.flysafety.ru/files/razdel2.pdf (Reference date: 13.10.15).

4. Status of safety in civil aviation of the States Parties of the agreement on civil aviation and the use of airspace over a ten year period (1992-200). Report of the Interstate Aviation Committee. URL: http://www.mak.ru/russian/info/doclad_bp/1992-2001/doklad_za_19922001_godi.html (Reference date: 13.10.15).

5. Friedman AE Fundamentals of metrology. Modern course. SPb, 2008; 284 p. 
6. Guidance on information support of the automated system to ensure the safety of aircraft of the Russian Federation Civil Aviation (ASOBP). M.: OOO "Aeronautical Consulting Agency", 2002; 191 p.

7. Maxwell JK. Understanding the gas dynamics theory. Phil. Mag., 1860; 19: 19-32.

8. Maxwell JK. Understanding the gas dynamics theory. Phil. Mag., 1860; 20: 21-37.

9. Bavrin II. A Short Course of Higher Mathematics for Chemical, Biological and Medical Majors. M.: Fizmatlit, 2003.

10. Hastings N, Peacock J. Handbook of statistical distributions, ed. by A. Zvonkina. M.: Statistika, 1880; 95 p. 